\title{
Milk, flavoured milk products and caries
}

R. S. Levine, '

\begin{abstract}
The consumption of flavoured milk increased by $50 \%$ between 1992 and 1999 and dental health educators need to know if these and other sugar and fruit juice sweetened milk products, such as fruit yoghurts, are acceptable as snack items. Available evidence suggests that their cariogenicity is negligible to low and consumed in moderation they are a preferable alternative to similarly sweetened soft drinks.
\end{abstract}

M ilk is of major nutritional value, especially for children, being their main source of calcium. ${ }^{1}$ The consumption of milk products such as flavoured milks, milkshakes and yoghurts has increased, the annual consumption of flavoured milks growing from 56 to 84 million litres between 1992 and $1999^{2}$ and the question of the cariogenicity of such products, while complex, must be addressed. This paper aims only to give a brief overview.

\section{Milk}

Cow's milk contains about $4-5 \%$ of the disaccharide lactose which in aqueous solution is metabolised by plaque organisms to organic acids with a consequent fall in plaque $\mathrm{pH}$, although to a lesser extent than with sucrose. However cow's milk produces a negligible fall in plaque $\mathrm{pH}$ in the mouth and the balance of evidence from animal and human studies suggests that cow's milk is non-cariogenic. ${ }^{3}$

The explanation for milk's apparent lack of cariogenicity appears to lie with its high buffering capacity and other components, including a high calcium and phosphate content. Milk proteins are adsorbed onto the enamel surface and may impede enamel demineralisation; the phosphoprotein casein is implicated in this effect. Milk fat adsorbs to the enamel surface and may have a protective role. Finally, milk enzymes may have a role in reducing the growth of acidogenic plaque bacteria. Cheese has been shown to help restore plaque $\mathrm{pH}$ after a sugar intake, probably

\footnotetext{
$1^{*}$ Independent Scientific Adviser and General Dental Practitioner, 370 Alwoodley Lane, Leeds LS17 7DN

${ }^{*}$ Correspondence to: Ronnie Levine email:ronnielevine@onetel.net.uk

REFEREED PAPER

Received 22.02.99; Accepted 23.11.00

(C) British Dental Journal 2001; 191: 20
}
In brief
- Unsweetened milk and milk-based products are safe for teeth, as are artificially sweetened products.
- Sugar and fruit juice sweetened milk products consumed in moderation are a preferable alternative to similarly sweetened soft drinks.
- The practice of adding sugar to milk should be discouraged.

caused by effective saliva stimulation, together with the effect of its milk components. The consensus of opinion is that milk and milk products, if unsweetened by added sugars, are safe and possibly beneficial for teeth. ${ }^{1}$

\section{Sugar-sweetened milk and milk products}

The increasing consumption of sugarsweetened milk products raises the question of their cariogenicity, however most available evidence comes from laboratory research. A study on rats showed an increase in cariogenicity with only $2 \%$ sucrose added to milk ${ }^{4}$ and an intra-oral plaque study found an increase in plaque acid production with $5 \%$ sucrose in milk. ${ }^{5}$ However another intra-oral study assessing demineralisation of bovine enamel slabs showed no effect from $5 \%$ sucrose in milk, but pronounced demineralisation with 5\% sucrose in water. ${ }^{6}$ In a human study, rinsing with $10 \%$ sucrose in milk produced similar plaque acidity to $10 \%$ sucrose in water and significantly more than with plain milk. ${ }^{7}$ In the only relevant clinical trial, institutionalised young people received a daily supplement of $0.45 \mathrm{~L}$ of chocolate milk (5\% sucrose) for 2 years, producing a small but non-significant increase in caries compared with a plain milk supplement. ${ }^{8}$ Flavoured milks, milkshakes and sugar-sweetened yoghurts typically contain $5 \%$ or more of added sugars. The low intrinsic $\mathrm{pH}$ found in many yoghurts may interfere with plaque glycolysis of sugars and one study found yoghurt with 5\% sucrose to have low cariogenic potential. 9

There are few reports on the cariogenicity of specific sweetened milk products and since testing procedures vary, generalisations must be guarded. However, it would appear from the available evidence that products containing about $5 \%$ added sugars have a negligible or low cariogenic potential which is likely to be less than that of aqueous sugar-sweetened beverages, of which the total UK consumption in 1999 was 11,000 million litres, under 16-year-olds consuming $60 \% .{ }^{10}$ The practice of adding sugar to milk should be discouraged. More research on the cariogenic potential of milk-based products is needed as they form an important part of our diet.

\section{Conclusion}

Because sweetened milk products have a nutritional value and may not be consumed by children as frequently as other sugarsweetened beverages, the cariogenic load produced in most individuals is likely to be low and their occasional consumption should be accepted.

1 Rugg-Gunn A J. Nutrition and dental health. Chapter 9. Oxford: Oxford University Press, 1993

2 National Dairy Council, London, 2000.

3 Jenkins G N, Ferguson D B. Milk and dental caries. Br Dent J 1966; 120: 472-477.

4 Bowen W H, Pearson S K. Effect of milk on cariogenesis. Caries Res 1993; 27: 461-466.

5 Thompson M E, Dever J G, Pearce E I F. Intraoral testing of flavoured sweetened milk. $N Z$ Dent J 1984; 86: 44-46.

6 Mor B M, McDougall W A. Effects of milk on $\mathrm{pH}$ of plaque and salivary sediment and the oral clearance of milk. Caries Res 1977; 11: 223-230.

7 Moynihan P J et al. Effect of glucose polymers in water, milk and a milk substitute on plaque $\mathrm{pH}$ in vitro. Int J Pediatr Dent 1996; 6: 19-24.

8 Dunning J M, Hodge A T. Influence of cocoa in milk on dental caries incidence. J Dent Res 1971; 50: 854-859.

9 Mundorff S A et al. Cariogenic potential of foods 1. Caries in the rat model. Caries Res 1990; 24: 344-355.

10 UK market review. Reading: Tate \& Lyle Speciality Sweeteners, 2000. 